\title{
Fast Multifrequency Measurement of Nonlinear Conductance
}

\author{
Riccardo Borgani, ${ }^{1,{ }^{*}}$ Mojtaba Gilzad Kohan, ${ }^{2}$ Alberto Vomiero, ${ }^{2}$ and David B. Haviland ${ }^{1}$ \\ ${ }^{1}$ Nanostructure Physics, KTH Royal Institute of Technology, Stockholm SE-106 91, Sweden \\ ${ }^{2}$ Department of Engineering Sciences and Mathematics, Luleå University of Technology, Luleå SE-971 87, Sweden
}

(Received 17 December 2018; revised manuscript received 3 April 2019; published 19 April 2019)

\begin{abstract}
We describe a phase-coherent multifrequency lock-in measurement technique that uses the inverse Fourier transform to reconstruct the nonlinear current-voltage characteristic of a nanoscale junction. The method provides separation of the galvanic and displacement currents in the junction and easy cancellation of the parasitic displacement current from the measurement leads. These two features allow us to overcome traditional limitations imposed by the low conductance of the junction and the high capacitance of the leads, thus providing an increase in measurement speed of several orders of magnitude. We demonstrate the method in the context of conductive atomic force microscopy, acquiring current-voltage characteristics at every pixel while scanning at standard imaging speed.
\end{abstract}

DOI: 10.1103/PhysRevApplied.11.044062

\section{INTRODUCTION}

The sensitive measurement of small currents in nanometer-scale junctions is a central problem in modern experimental physics. Characterization of numerous materials and devices, in applications ranging from topological quantum computers [1,2] to energy harvesting and energy conversion [3-10], struggles with the same basic limitations imposed by the small measurement current and the large stray capacitance of the macroscopic leads. We describe how to circumvent these limitations using phasecoherent multifrequency lock-in measurement and inverse Fourier transform to achieve a dramatic increase in the speed of measurement, or alternatively, in the signal-tonoise ratio (SNR) at the same measurement speed. In addition, our frequency-domain approach allows active cancellation of parasitic current due to the lead capacitance and provides unambiguous separation of the galvanic and displacement currents flowing in the nanoscale junction.

One area where this improvement is particularly useful is scanning probe microscopy (SPM), where a measurement of the nonlinear current-voltage characteristic (IVC) is desired at each tip location. In scanning tunneling microscopy, the IVC allows mapping of the energy dependence of the local density of electronic states [11].

\footnotetext{
*borgani@kth.se
}

Published by the American Physical Society under the terms of the Creative Commons Attribution 4.0 International license. Further distribution of this work must maintain attribution to the author(s) and the published article's title, journal citation, and DOI.
In conductive atomic force microscopy (AFM) it can be used to map energy-conversion efficiency in photoactive nanocomposite materials [12]. Because of the aforementioned limitations, present-day SPM has two basic modes of operation. In imaging mode the current is measured while the surface is scanned with a constant tip-sample voltage, quickly generating an image but with only limited information. Multiple scans at different biases are required to get the full IVC, greatly increasing the measurement time and introducing problems due to instrument drift and tip wear. In spectroscopic mode the IVC is recorded at each tip position, but the voltage must be swept slowly so as to minimize displacement current in the parallel capacitance of the measurement leads. This large background current puts a limit on the achievable gain and sensitivity of current measurement, and the slow sweep greatly limits the speed of the scan, or equivalently, spatial resolution in a given measurement time. We demonstrate how to bridge the gap between these two modes of SPM, achieving complete electrical characterization at scanning speeds characteristic of the imaging mode.

Such characterization is important because the macroscopic device functionalities are strongly influenced by the nanometer-scale structure of heterojunctions (e.g., in solar cells $[13,14])$. Pioneering work in this general direction used high-speed acquisition to capture data while scanning at imaging speeds [15]. The large data sets [16] (several gigabytes per scan) were subsequently analyzed with advanced filtering methods based on statistical inference, separately reconstructing galvanic current and capacitance at each image pixel. However, this "big-data" approach is computationally expensive, requiring several hours of analysis on the fastest computers. 
In contrast, we take a deterministic (physical) approach that uses our knowledge of the periodicity and phase of the applied bias. Because we know the period of the drive waveform, a frequency-domain representation of the nonlinear response efficiently rejects noise and compresses data to a manageable size for storage. The inverse-fastFourier-transform (FFT) algorithm makes the analysis computationally efficient and easily performed in real time on a notebook computer. Apart from the physical model of the measurement, our reconstruction does not require assumptions about the functional form of the nonlinear galvanic current or the bias dependence of the junction capacitance.

Below we describe our method using conductive AFM as an example, but the general idea has broad applicability in many areas of experimental physics, where, with the recent development of multifrequency digital lock-in amplifiers, its implementation has been greatly simplified.

\section{THEORY}

Figure 1 shows a schematic representation of an SPM measurement and its equivalent circuit. When a timedependent bias voltage $V(t)$ is applied between the SPM tip and a conductive sample, the total current measured at the amplifier is

$$
\begin{aligned}
I & =G(V) V+C(V) \dot{V}+C_{P} \dot{V}+C_{C} \dot{V}_{C} \\
& \equiv I_{G}+I_{D}+I_{P}+I_{C} .
\end{aligned}
$$

We call the terms $I_{G}, I_{D}, I_{P}$, and $I_{C}$ the galvanic, displacement, parasitic, and compensation currents, respectively. The bias and its time derivative $\dot{V}$ are known signals. The conductance $G$ and capacitance $C$ between the tip and the

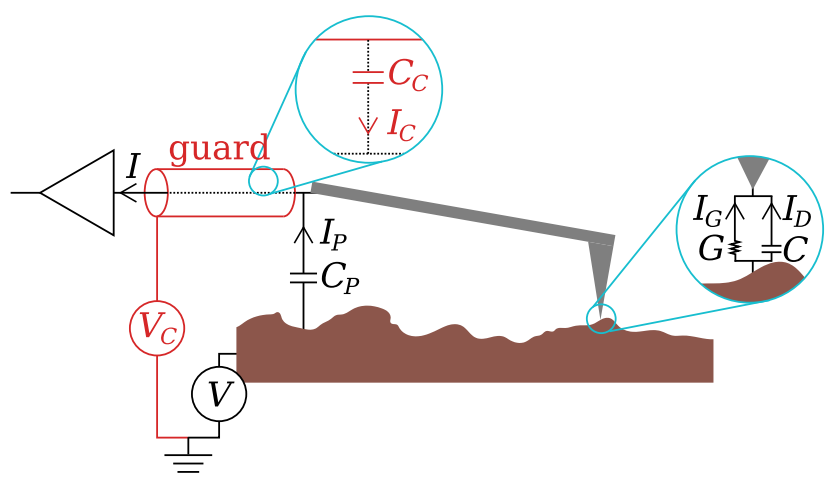

FIG. 1. Experimental setup and electrical connections. The conductance $G$ and the capacitance $C$ model the tip-sample junction, $C_{P}$ is the parasitic capacitance, $C_{C}$ is the capacitance of the coaxial cable, $V$ is the tip-sample bias voltage, $V_{C}$ is the compensation drive to the guard of the coaxial cable, and $I$ is the total current at the input of the transimpedance amplifier. The compensation circuit is shown in red. sample are the desired quantities in our physical model. We emphasize that both the conductance $G(V)$ and the capacitance $C(V)$ can be nonlinear functions of $V$. $C_{P}$ is a distributed parasitic capacitance in parallel with the junction, modeling the capacitive contributions of the probe body, cables, and measurement electronics. Good electrical design with proper guarding can minimize $C_{P}$. However, when current is measured in the subpicoampere range, the residual $I_{P}$ due to the unguarded $C_{P}$ can easily saturate the transimpedance amplifier, limiting its gain and thus sensitivity. We compensate for this parasitic current as follows.

With the tip lifted away from the sample surface (tens to hundreds of micrometers), the first two terms in Eq. (1) are both zero. The measured current is then $I_{\text {lift }}=C_{P} \dot{V}+$ $C_{C} \dot{V}_{C}$, where $V_{C}$ is applied to the guard. We obtain the value for $C_{P}$ by measuring $I_{\text {lift }}$ when applying a known $\dot{V}$ and keeping $V_{C}$ at zero, and similarly for $C_{C}$ :

$$
C_{P}=\left.\frac{I_{\text {lift }}}{\dot{V}}\right|_{V_{C}=0}, \quad C_{C}=\left.\frac{I_{\text {lift }}}{\dot{V}_{C}}\right|_{V=0} .
$$

Once $C_{P}$ is obtained from Eq. (2), the parasitic displacement current $I_{P}$ can be passively compensated for by subtracting it from the measured current. To not limit the amplifier gain, however, it is far better to actively compensate for the parasitic displacement current $I_{P}$ by nulling it before it reaches the amplifier: with $C_{C}$ from Eq. (2), we apply a $V_{C}$ that exactly cancels the contribution from the parasitic capacitance, $V_{C}(t)=-\left(C_{C} / C_{P}\right) V(t)$. Because $C_{P}$ is at the millimeter to meter scale (probe holder, cables, and measurement electronics), its value does not change significantly between the lifted and scanning positions of the SPM tip. The compensation voltage $V_{C}$ is therefore constant while scanning.

To measure the nonlinear galvanic and displacement currents, we apply a time-dependent sample bias of the form $V(t)=V_{\mathrm{ac}} \cos \omega_{1} t$. Assuming the junction conductance and capacitance are analytic functions of the voltage, they both share the same time periodicity as $V$ and can therefore be written as a Fourier series: $G[V(t)]=$ $\sum_{m} g_{m} \cos m \omega_{1} t$ and $C[V(t)]=\sum_{m} c_{m} \cos m \omega_{1} t$. The two components of the current are

$$
\begin{gathered}
I_{G}(t)=G(t) V_{\mathrm{ac}} \cos \omega_{1} t=\sum_{k=0}^{+\infty} I_{G_{k}} \cos k \omega_{1} t, \\
I_{D}(t)=-C(t) \omega_{1} V_{\mathrm{ac}} \sin \omega_{1} t=\sum_{k=0}^{+\infty} I_{D_{k}} \sin k \omega_{1} t,
\end{gathered}
$$

where the $I_{G_{k}}$ and $I_{D_{k}}$ are real constants. Equations (3) and (4) show that the two current contributions are easily distinguishable: the galvanic current is in phase with $V$, 
and the displacement current is in phase with $\dot{V}$. We can therefore obtain the galvanic and displacement currents from the real part and the imaginary part, respectively, of the Fourier transform of the measured compensated current:

$$
\hat{I}_{G}(\omega)=\operatorname{Re}[\hat{I}(\omega)], \quad \hat{I}_{D}(\omega)=i \operatorname{Im}[\hat{I}(\omega)]
$$

\section{EXPERIMENTAL RESULTS}

Figure 2 demonstrates the technique with conductive AFM, measuring the IVC between a Pt-coated AFM tip and an organic solar cell consisting of a TQ1-T10 polymer blend on a polyethylenimine-modified poly $(3,4-$ ethylenedioxythiophene) polystyrene sulfonate transparent electrode. The Fourier components of the current $\hat{I}(\omega)=$ $\hat{I}_{G}+i \hat{I}_{D}$ are measured simultaneously at 31 harmonics of $\omega_{1}$ [Fig. 2(a)] with a multifrequency lock-in amplifier (MLA) [17]. Using the inverse Fourier transform, we obtain the total current $I$ (blue), galvanic current $I_{G}$ (orange) and displacement current $I_{D}$ (green), as shown in Fig. 2(b).

In Fig. 2(c) we plot $I_{G}(t)$ versus the applied $V(t)$ to obtain the IVC, free of any capacitive contribution (orange curve). The organic photovoltaic material in Fig. 2 is measured under white-light illumination, where the galvanic IVC shows the typical photodiode response, with an offset current at zero applied voltage (short-circuit current $I_{\mathrm{SC}}$ ) and a finite voltage required to obtain zero current (opencircuit voltage $\left.V_{\mathrm{OC}}\right)$. For comparison, we also plot the IVC that one would obtain from the total current (blue curve); that is, without separation of the galvanic and displacement contributions. The junction capacitance produces a big hysteresis loop that completely masks these important features of the galvanic IVC. In standard conductive AFM, this hysteresis loop would be even larger as the parasitic capacitance $C_{P}$, eliminated by active cancellation, is orders of magnitude larger than the junction capacitance $C$.

The ability to separate the junction's displacement current from its galvanic current allows us to plot the junction capacitance $C(V)=I_{D}(t) / \dot{V}(t)$ versus $V(t)$, shown by the green curve in Fig. 2(c). This capacitance is ideally that of the junction itself as the large parallel capacitance is removed when one is compensating for $I_{P}$. However, some residual parallel capacitance local to the tip may be included, depending on the exact geometry of the tip and surface when one is nulling $I_{P}$. The ability to measure the voltage dependence of the tip-sample capacitance in parallel with the galvanic IVC is an exciting feature of this technique, which may be useful to investigate the screening length in two-dimensional electron gases [18] or quantum capacitance in low-dimensional devices [19].

Acquiring the full IVC at every pixel of an AFM scan allows the rapid mapping of interesting electrical properties, such as $I_{\mathrm{SC}}$ and $V_{\mathrm{OC}}$. Because of the computational efficiency of the reconstruction, quantities such as the maximum-power point or fill factor can be evaluated and displayed in real time during the scan (see the maps in Appendix D), and these quantities can be correlated with the topography to investigate structure-property relationships. As a demonstration of the resolution enabled by this measurement method, we analyze another sample consisting of a $\mathrm{CuO}_{2} / \mathrm{ZnO}$ thin-film $p$ - $n$ junction deposited on
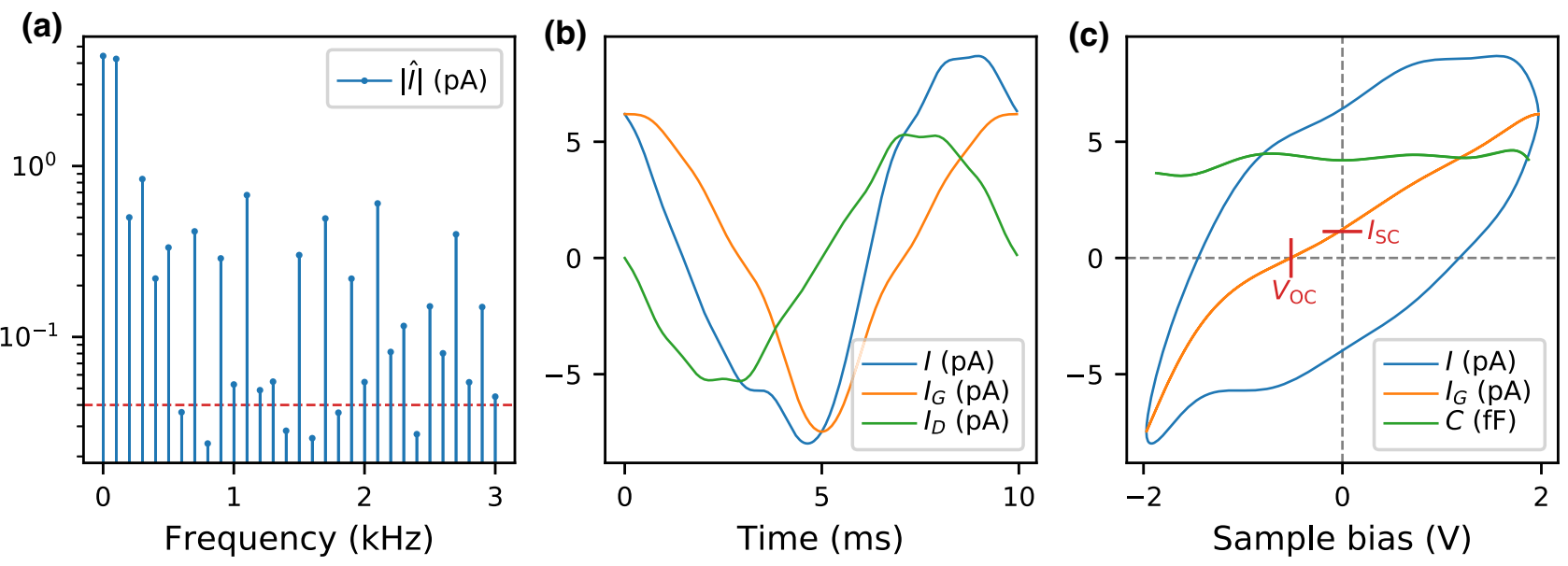

FIG. 2. Current-voltage characteristics on a photoactive polymer blend. Measurement under white-light illumination. (a) Amplitude of the measured current at 31 frequencies; the phase is also measured but is not shown. The dashed red line is the noise level at the measurement bandwidth $\Delta \omega /(2 \pi)=100 \mathrm{~Hz}$. (b) Total, galvanic, and displacement currents as a function of time obtained from the current spectrum by inverse Fourier transform of the expressions in Eq. (5). (c) Reconstructed currents and junction capacitance versus voltage. The loop in the total current $I(V)$ is due to the junction capacitance. The galvanic current $I_{G}(V)$ does not show such a loop. The junction capacitance $C$ is nearly constant, as expected. 


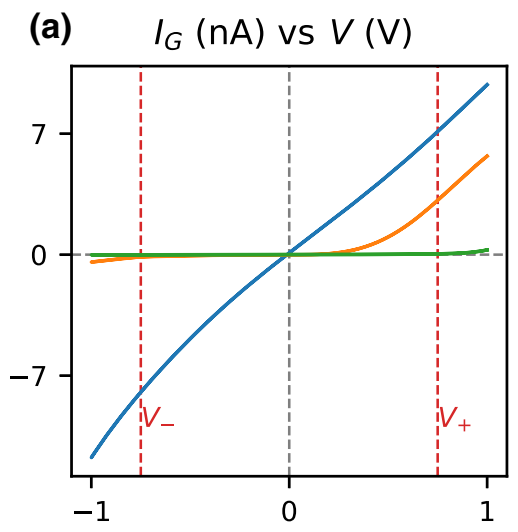

(b)

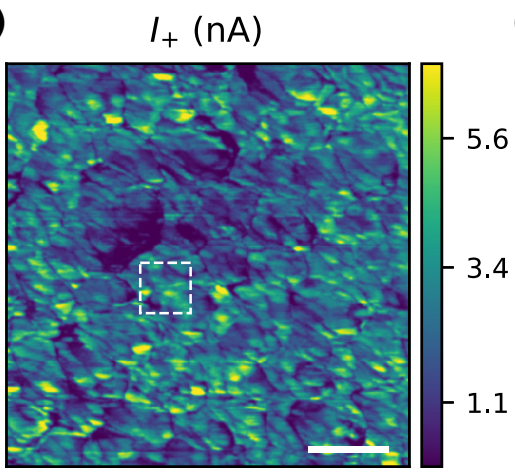

(e)

(d)

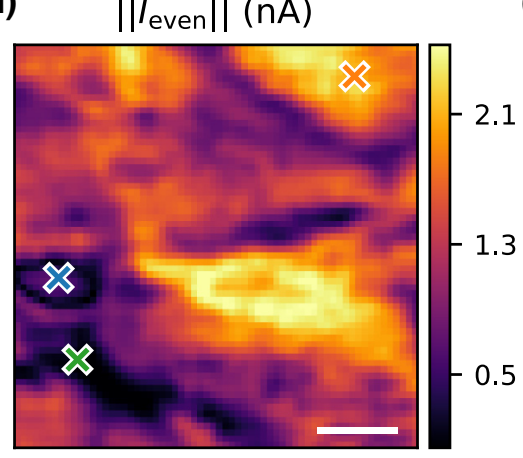

(c)

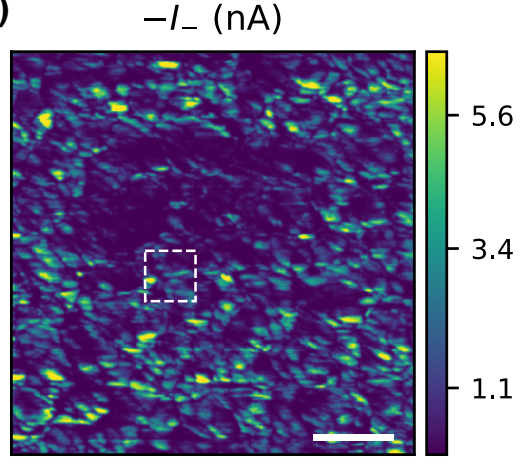

(f)

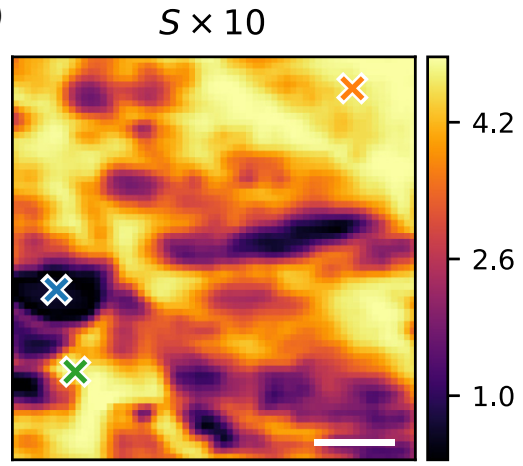

FIG. 3. Measurements on a thin-film all-oxide $p$ - $n$ junction. (a) Representative IVCs at different locations of the sample. Maps of (b) current $I_{+}$at forward bias $V_{+}=0.75 \mathrm{~V}$, (c) current $I_{-}$at reverse bias $V_{-}=-0.75 \mathrm{~V}$, (d) even-symmetry current $\left\|I_{\text {even }}\right\|$, (e) odd-symmetry current $\left\|I_{\text {odd }}\right\|$, and (f) symmetry parameter $S=\left\|I_{\text {even }}\right\| /\left(\left\|I_{\text {even }}\right\|+\left\|I_{\text {odd }}\right\|\right)$. The maps in (d)-(f) are on an enlarged area marked by the dashed white boxes in (b),(c). The white scale bars are $200 \mathrm{~nm}$ in (b),(c) and $25 \mathrm{~nm}$ in (d)-(f). The crosses in (d)-(f) mark the positions where the IVCs of corresponding colors are acquired.

a fluorine-doped tin oxide conducting glass. Such composite oxide materials find application in sensing [20], energy conversion [21,22], and lighting [23], and their nanoscale characterization is needed to understand their functionality.

Figure 3(a) shows that the junction of the two semiconductors presents a typical diodelike IVC, with current flowing only for positive applied bias (orange curve). However, some areas of the sample show a symmetric IVC (blue curve), indicating damage in the $\mathrm{CuO}_{2}$ or a resistive phase of the composite material. Other areas show zero current in both bias directions (green curve), indicating a much smaller area of contact between the tip and the sample, or perhaps an insulating phase of material. To analyze the spatial distribution of these three classes of IVC, we plot the current $I_{+}$at fixed forward bias $V_{+}=$ $0.75 \mathrm{~V}$ and the current $I_{-}$at fixed reversed bias $V_{-}=$ $-0.75 \mathrm{~V}$. The $I_{+}$and $I_{-}$maps can be obtained with standard conductive AFM by performing multiple scans with different applied bias $V_{+}$or $V_{-}$. Here, however, current maps for any bias $-V_{\mathrm{ac}}<V<V_{\mathrm{ac}}$ can be calculated from the measured data as the whole IVC is acquired in a single scan.
In the forward-bias image [Fig. 3(b)] both diodelike and resistorlike areas have high current and appear as bright spots, while areas with no current are dark. In the reversebias image [Fig. 3(c)], the resistorlike areas stand out as bright spots, while the diodelike and no-current areas remain dark. To more precisely distinguish the diodelike areas, we focus on a small region of these images (dashed white lines) and we analyze the symmetry properties of the acquired IVCs: resistorlike curves have a clear odd symmetry around zero [Fig. 3(e)], and regions with diodelike curves have a stronger even-symmetry component [Fig. 3(d)]. The two images are combined in Fig. 3(f) with use of the symmetry parameter $S$, which is 0 for a purely odd curve and 1 for a purely even curve (for more details, see Appendix B).

The fine detail in these images is possible due the very high density of the measured IVCs, such as those in Fig. 3(a), which are acquired at 1000 pixels/s, yielding a trace-retrace image with $512 \times 512$ resolution $(524,288$ IVCs, file size $170 \mathrm{MB}$ ) in less than $9 \mathrm{~min}$. With conductive AFM in spectroscopic mode, one would typically sweep the bias in 1-10 s, giving a total measurement time of 6-60 days for the same spatial resolution. The analysis 
of the full image requires about $4 \mathrm{~s}$ on a laptop computer, and it is therefore easily performed in real time (i.e., while the AFM is scanning).

The pixel rate is set by the measurement bandwidth $\Delta \omega$, which has a maximum value of $\omega_{1}$. The choice of $\omega_{1}$ in turn determines how many harmonics can be resolved within the bandwidth of the transimpedance amplifier, and thereby the sharpness of the features in the reconstructed IVC (see the detailed discussion in Appendix C). The measurement bandwidth also sets the signal-to-noise ratio (noise proportional to $\sqrt{\Delta \omega}$ ). In Fig. 2(a) the noise level is $0.04 \mathrm{pA}(4 \mathrm{fA} / \sqrt{\mathrm{Hz}})$, which allows $n=10$ harmonics within the amplifier bandwidth for $\omega_{1} / 2 \pi=100 \mathrm{~Hz}$. In Fig. 3 the noise level is $1.3 \mathrm{pA}(40 \mathrm{fA} / \sqrt{\mathrm{Hz}})$, with $\omega_{1} / 2 \pi=1 \mathrm{kHz}$ and $n=50$.

For a given SNR, and therefore a given measurement time $T_{M}=2 \pi / \Delta \omega$, our frequency-multiplexed method offers a clear speedup over traditional time-domain methods. A time-domain measurement of the IVC would apply a certain dc bias, average the measured current for a time $T_{M}$, change the applied bias, and repeat this procedure until $n$ points of the IVC are acquired in a total time $n T_{M}$. In our method, a MLA acquires $n \approx 32$ harmonics of $\omega_{1}$ in the same time $T_{M}$ (in parallel), obtaining $n$ data points to describe the nonlinear IVC. We thus achieve a factor-of- $n$ increase in speed at the same SNR, or alternatively a factor-of- $\sqrt{n}$ increase in the SNR for the same total measurement time. This increase in speed comes in addition to that enabled by cancellation and separation of displacement currents.

An important consideration with the method described here is that $\omega_{1}$, its harmonics, and the sampling frequency used by the MLA must all be integer multiples of the measurement bandwidth $\Delta \omega$. This "tuning" eliminates Fourier leakage in the harmonic spectrum, ensuring that the amplitude and phase of all the harmonics are measured coherently. The information about the nonlinear character of the IVC is then coded in the harmonics and revealed by simple inverse Fourier transform.

\section{CONCLUSIONS}

We describe and demonstrate a measurement paradigm for capturing nonlinear current-voltage characteristics from weak and noisy signals. Using one stable reference oscillation for phase-sensitive detection of many harmonics, we achieve frequency-domain multiplexing of the information contained in the nonlinear IVC. Another important advantage of our frequency-domain approach is that we can easily extract the separate contributions of the galvanic and displacement currents in the measured total current. The frequency-domain data are an optimally compressed representation of the nonlinear response and it is computationally efficient to reconstruct the IVC with use of the inverse-FFT algorithm. The frequency-domain approach also provides simple cancellation of the large parasitic current due to the stray capacitance of the leads, allowing larger gain without saturation of the current amplifier. Together these advantages allow greatly enhanced measurement speed, compact data storage, and real-time feedback during measurement.

We use scanning probe microscopy to demonstrate the power of the method, reconstructing the full IVC at every pixel of a conductive-AFM image, without compromising the scanning speed. With the IVC at every pixel, we construct a posteriori images that highlight the interesting figures of merit for electric transport. With standard conductive AFM, the set of bias voltages is decided a priori, before multiple scans are performed. During the long measurement, instrument drift and tip wear inhibit reliable correlation between the tip position and the measured electrical properties. The multifrequency approach provides a general solution to a very-general and very-common problem in nanotechnology, and its easy implementation has recently been made possible with the advent of tuned multifrequency digital lock-in amplifiers.

\section{ACKNOWLEDGMENTS}

The authors thank O. Inganäs and Y. Xia for providing the organic-solar-cell sample. D.B.H. and R.B. acknowledge financial support from the Swedish Research Council (VR) and the Knut and Alice Wallenberg Foundation. A.V. and M.G.K. acknowledge financial support from the Kempe Foundation and the Knut and Alice Wallenberg Foundation.

\section{APPENDIX A: INSTRUMENTATION}

The current flowing through the AFM tip $I$ is converted to voltage by a DLPCA-200 variable-gain, low-noise current amplifier (FEMTO Messtechnik GmbH). The voltage is then sampled by a multifrequency lock-in amplifier (Intermodulation Products $\mathrm{AB}$ ), and the real and imaginary parts of 32 frequency components $I_{G_{k}}$ and $I_{D_{k}}$ are read via an Ethernet connection by a computer for analysis. Two output ports on the MLA are used to apply the sample bias $V$ and the compensation voltage $V_{C}$. The measurement and analysis routines are implemented in the scripting interface of the MLA control software. They are written in PYTHON and make use of the inverse fast Fourier transform [24] and other routines in the SciPy and NumPy libraries [25].

The measurements on the organic-solar-cell sample are performed with a NanoWizard ULTRA Speed AFM (JPK Instruments AG) mounted on an inverted optical microscope (Nikon Corporation). The microscope objective is used to focus the light from a white-light-emitting diode onto a small spot around the area scanned. The illumination by the light-emitting diode is alternately switched 

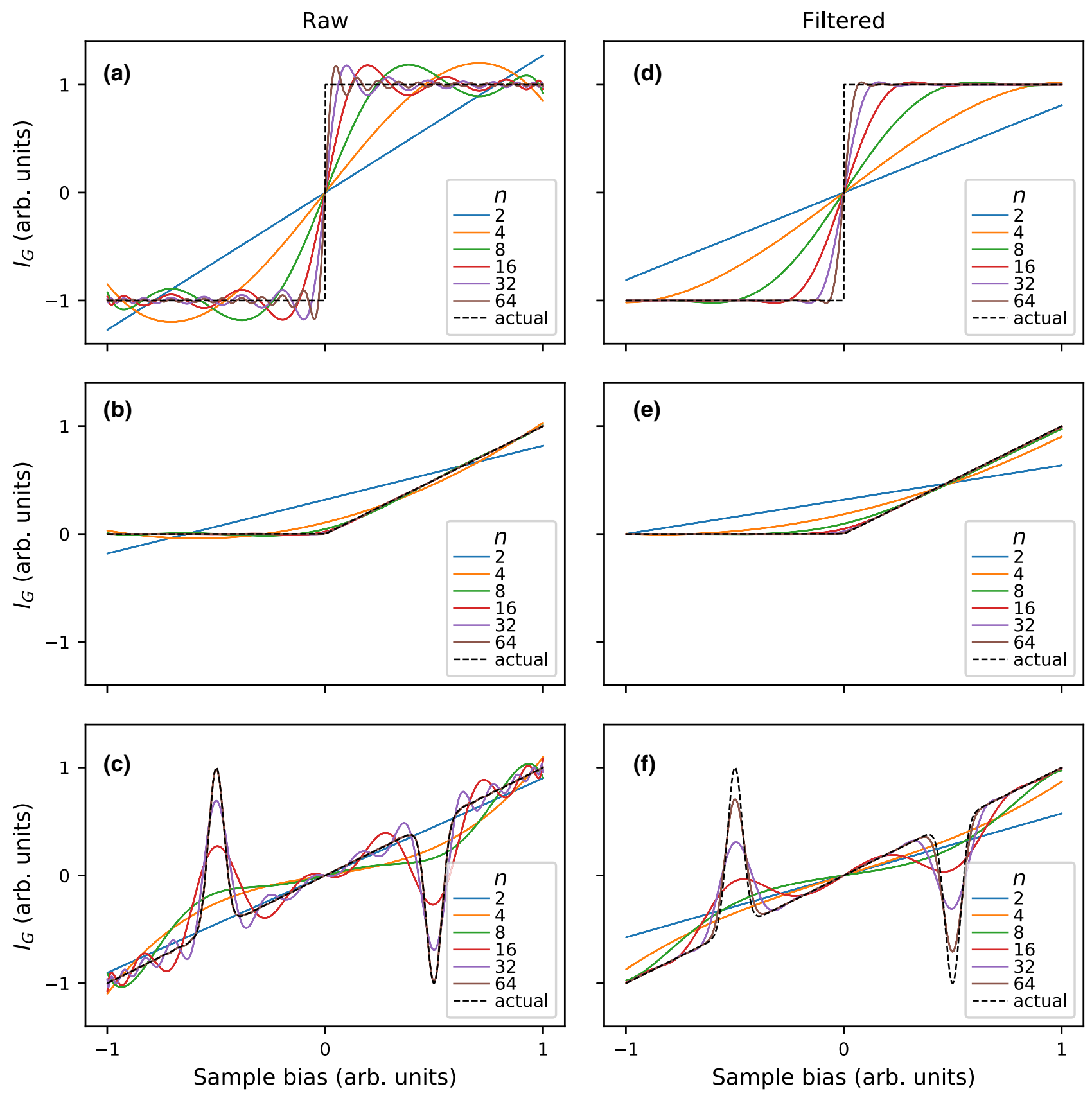

FIG. 4. IVC reconstruction with a limited number of harmonics. Simulation of IVCs with sharp features (dashed black lines) in analogy to (a),(d) Josephson junctions, (b),(e) Coulomb-blockade junctions, and (c),(f) resonant-tunneling diodes. In the raw curves (a) (c) the spectrum is truncated at $\omega=n \omega_{1}$, mimicking a limited number of demodulators. In the filtered curves (d)-(f) the truncation is smoothed in the frequency domain according to Eq. (C1), suppressing the ringing artifacts and simulating a limited available bandwidth.

on and off during the scanning trace and retrace (approximately $1 \mathrm{~Hz}$ ) to compare the electrical response of the material under light and dark conditions. The measurements on the $\mathrm{CuO}_{2} / \mathrm{ZnO}$ sample are performed with a Dimension Icon AFM (Bruker Corporation). In both cases, the AFM is operated in contact mode with an RMN12PT400B cantilever (Bruker, nominal spring constant $0.3 \mathrm{~N} / \mathrm{m})$.
In principle, the current amplifier built into the AFM can be used instead of the external DLPCA-200 amplifier. However, the external amplifier used in this study allows easier access to the input guard, and therefore easier implementation of the compensation routine. Similarly, one could perform the experiments described herein without the MLA, by using a high-speed sampling card synchronized with two signal generators. Care must be 

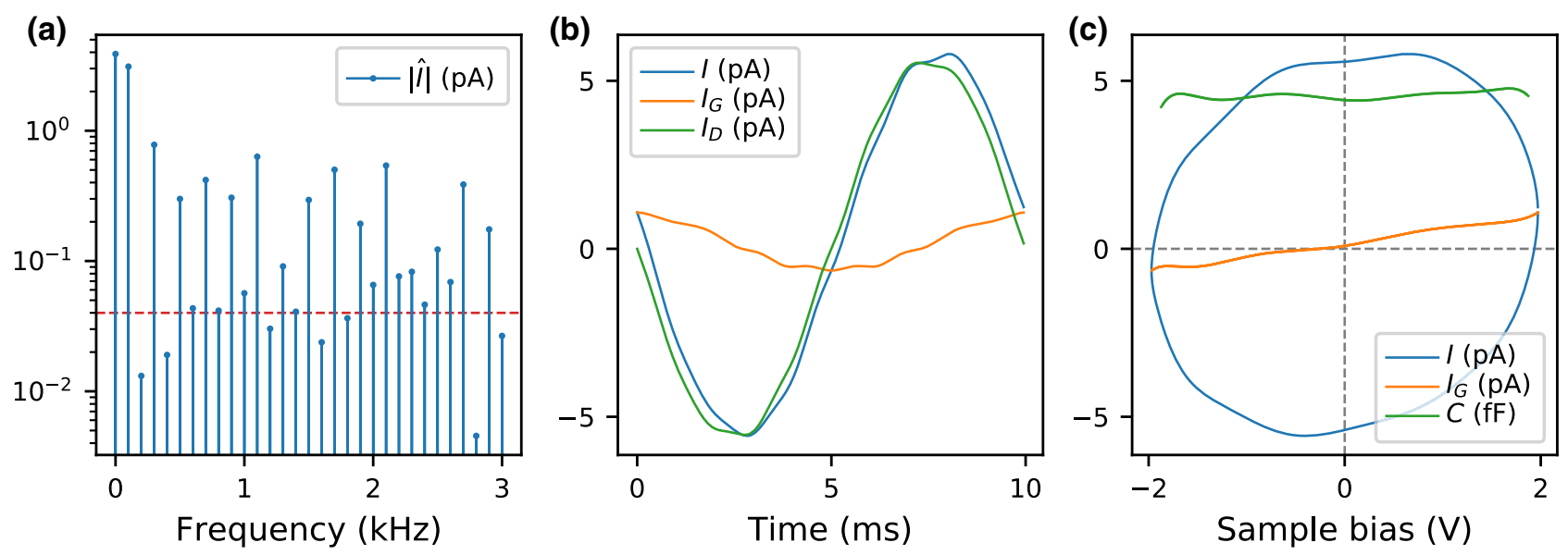

FIG. 5. Current-voltage characteristics of a photoactive polymer blend. Measurement in dark conditions. (a) Amplitude of the measured current at 31 frequencies; the phase is also measured but is not shown. The dashed red line is the calibrated noise level. (b) Total, galvanic, and displacement currents as a function of time obtained from the current spectrum by inverse Fourier transform of the expressions in Eq. (5). (c) Reconstructed currents and junction capacitance versus voltage. The loop in the total current $I(V)$ is due to the junction capacitance. The galvanic current $I_{G}(V)$ does not show such a loop. The junction capacitance $C$ is nearly constant, as expected.

taken that all the frequencies of interest are tuned to minimize Fourier leakage, and the analysis would need to be performed entirely on the computer (limiting the real-time capabilities of the analysis).

\section{APPENDIX B: SYMMETRY OF THE IVC}

An arbitrary curve can be decomposed into an evensymmetry and an odd-symmetry curve around zero. For (a)

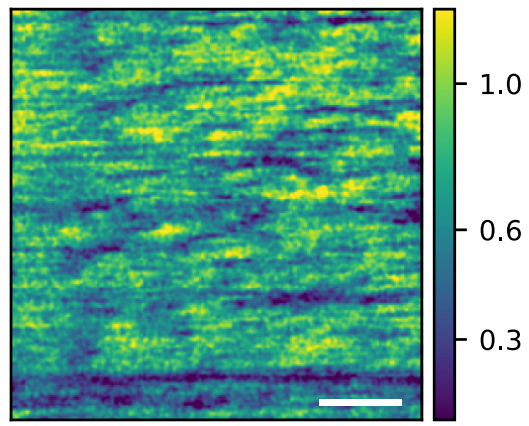

(d)

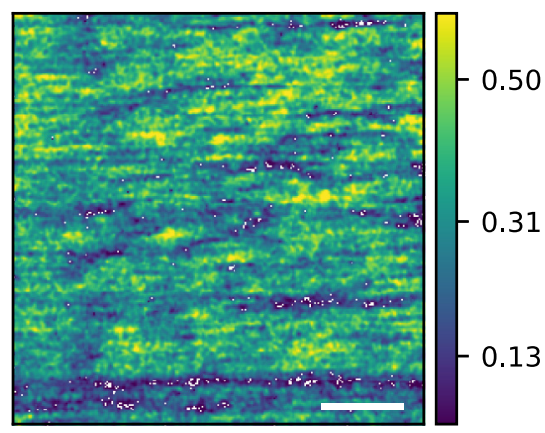

(b) $\quad-V_{\mathrm{OC}}(\mathrm{mV})$

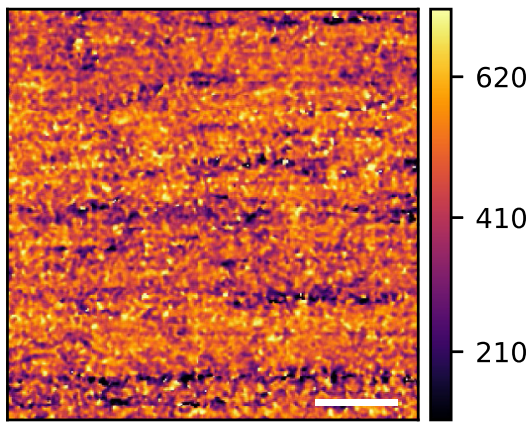

(e) $\quad-V_{\mathrm{MP}}(\mathrm{mV})$

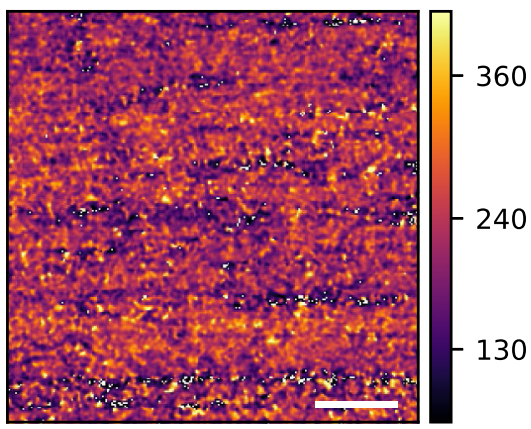

(c) $\quad I_{\mathrm{RV}}(\mathrm{pA})$

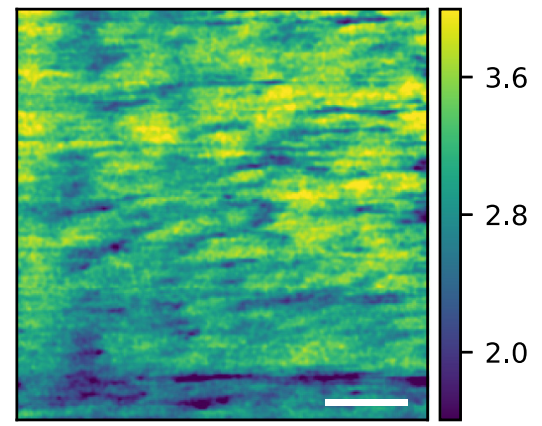

(f)

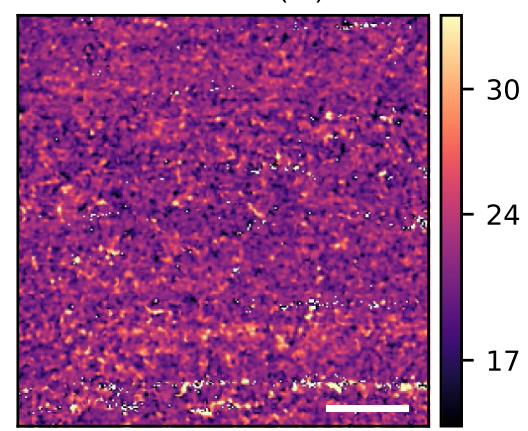

FIG. 6. Parameter maps for a photoactive polymer blend. Maps and histograms of (a) short-circuit current $I_{\mathrm{SC}}$, (b) open-circuit voltage $V_{\mathrm{OC}}$, (c) reverse-bias current $I_{\mathrm{RV}}$, (d) current at maximum-power point $I_{\mathrm{MP}}$, (e) voltage at maximum-power point $V_{\mathrm{MP}}$, and (f) fill factor $\left(I_{\mathrm{MP}} V_{\mathrm{MP}} / I_{\mathrm{SC}} V_{\mathrm{OC}}\right)$. The white scale bars are $200 \mathrm{~nm}$. 
our IVC

$$
\begin{gathered}
I_{G}(V)=I_{\mathrm{even}}(V)+I_{\mathrm{odd}}(V), \\
I_{\text {even }}(V)=\frac{1}{2}\left[I_{G}(V)+I_{G}(-V)\right], \\
I_{\mathrm{odd}}(V)=\frac{1}{2}\left[I_{G}(V)-I_{G}(-V)\right] .
\end{gathered}
$$

We then quantify the magnitude of each contribution by using the norm

$$
\|I\|^{2}=\frac{1}{2 V_{\mathrm{ac}}} \int_{-V_{\mathrm{ac}}}^{V_{\mathrm{ac}}}|I(V)|^{2} \mathrm{~d} V
$$

and the symmetry parameter defined as

$$
S=\frac{\left\|I_{\text {even }}\right\|}{\left\|I_{\text {even }}\right\|+\left\|I_{\text {odd }}\right\|} .
$$

$S$ is 0 for a purely odd curve, 1 for a purely even curve, and between 0 and 1 for any other curve.

\section{APPENDIX C: RECONSTRUCTION OF SHARP FEATURES}

The IVCs shown in Figs. 2(c) and 3(a) are relatively smooth (i.e., they do not present sharp features and are thus easily reconstructed with a limited number of harmonics). However, many experiments are characterized by sharp features in the IVC (e.g., Josephson junctions, Coulombblockade junctions, and resonant-tunneling diodes). The simulations in Fig. 4 show that in such cases many frequency components are needed to reconstruct the actual IVC (dashed black line).

In the "raw" curves [Figs. 4(a)-4(c)] the frequency spectra of the galvanic current are truncated at $n$ frequency components $(\omega=0$ included $)$, simulating the effect of a limited number of available demodulators in the MLA. The reconstructed IVC approaches the actual curve as $n$ increases, but ringing artifacts persist due to the sharp truncation of the frequency spectrum, in analogy to the Gibbs phenomenon. These artifacts are strongly suppressed in the "filtered" curves [Figs. 4(d)-4(f)], where the current spectra are smoothly limited to $n$ components with a window inspired by the $\sigma$ approximation:

$$
\hat{W}(k)=\left(\operatorname{sinc} \frac{k}{n}\right)^{\sigma},
$$

where $k=\omega / \omega_{1}$ and $\sigma=1$ is a smoothing parameter. The filtered curves more closely mimic the case where $n$ is limited by the bandwidth of the transimpedance amplifier. (a) Height $(\mathrm{nm})$

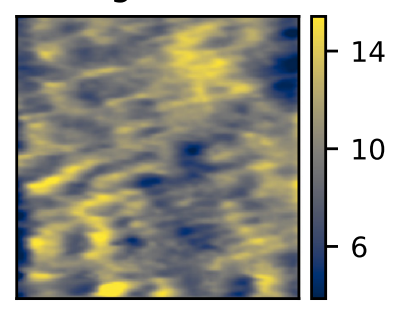

(b) Height $(\mathrm{nm})$

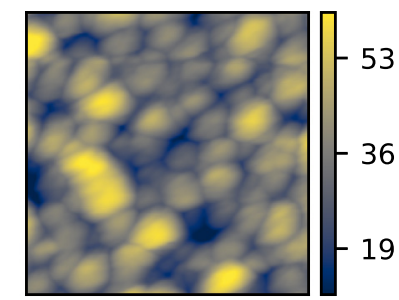

FIG. 7. Height images. (a) Organic photovoltaic material in Figs. 2, 5, and 6. (b) Thin-film all-oxide $p-n$ junction in Fig. 3. The white scale bars are $200 \mathrm{~nm}$.

\section{APPENDIX D: MAPPING OF MATERIAL PROPERTIES OF AN ORGANIC PHOTOVOLTAIC MATERIAL}

Figure 5 shows the current measured on the organic photovoltaic material in dark conditions, for comparison with the measurement under illumination shown in Fig. 2. The displacement current (green line) and thus the capacitance are not affected by the change in illumination. The galvanic current, on the other hand, significantly decreases in dark conditions: the diodelike shape of the IVC disappears together with $I_{\mathrm{SC}}$ and $V_{\mathrm{OC}}$, and only a high residual resistance is visible.

Figure 6 shows the reconstructed values of $I_{\mathrm{SC}}, V_{\mathrm{OC}}$, and the current $I_{\mathrm{RV}}$ measured at a reverse bias of $1 \mathrm{~V}$. Because of the computational efficiency of the inverse FFT, the analysis of the entire scan takes less than $1 \mathrm{~s}$ on a notebook computer and can therefore be performed in real time, while one is scanning with the AFM. In all panels showing current [Figs. 6(a), 6(c), and 6(d)], a vertical line and a horizontal line are visible on the left and at the bottom, respectively. These low-current features are an indication of damage to the sample material caused by a previous scan with a too-high contact-mode set point.

\section{APPENDIX E: HEIGHT IMAGES}

Figure 7 shows the AFM height images obtained from the quasistatic mode (contact mode) feedback (i.e., at constant cantilever deflection).

[1] R. M. Lutchyn, E. P. A. M. Bakkers, L. P. Kouwenhoven, P. Krogstrup, C. M. Marcus, and Y. Oreg, Majorana zero modes in superconductor-semiconductor heterostructures, Nat. Rev. Mater. 3, 52 (2018).

[2] Önder Gül, Hao Zhang, Jouri D. S. Bommer, Michiel W. A. de Moor, Diana Car, Sébastien R. Plissard, Erik P. A. M. Bakkers, Attila Geresdi, Kenji Watanabe, Takashi Taniguchi, and Leo P. Kouwenhoven, Ballistic Majorana nanowire devices, Nat. Nanotechnol. 13, 192 (2018). 
[3] Mengwei Si, Chun-Jung Su, Chunsheng Jiang, Nathan J. Conrad, Hong Zhou, Kerry D. Maize, Gang Qiu, ChienTing Wu, Ali Shakouri, Muhammad A. Alam, and Peide D. Ye, Steep-slope hysteresis-free negative capacitance $\mathrm{MoS}_{2}$ transistors, Nat. Nanotechnol. 13, 24 (2018).

[4] Pedram Ghamgosar, Federica Rigoni, Shujie You, Illia Dobryden, Mojtaba Gilzad Kohan, Anna Lucia Pellegrino, Isabella Concina, Nils Almqvist, Graziella Malandrino, and Alberto Vomiero, $\mathrm{ZnO}-\mathrm{Cu}_{2} \mathrm{O}$ core-shell nanowires as stable and fast response photodetectors, Nano Energy 51, 308 (2018).

[5] Anja Wedig, Michael Luebben, Deok-Yong Cho, Marco Moors, Katharina Skaja, Vikas Rana, Tsuyoshi Hasegawa, Kiran K. Adepalli, Bilge Yildiz, Rainer Waser, and Ilia Valov, Nanoscale cation motion in TaOx, HfOx and TiOx memristivesystems, Nat. Nanotechnol. 11, 67 (2015).

[6] Rüdiger Berger, Hans Jürgen Butt, Maria B. Retschke, and Stefan A. L. Weber, Electrical modes in scanning probe microscopy, Macromol. Rapid Commun. 30, 1167 (2009).

[7] Jun Liu, Ankur Goswami, Keren Jiang, Faheem Khan, Seokbeom Kim, Ryan McGee, Zhi Li, Zhiyu Hu, Jungchul Lee, and Thomas Thundat, Direct-current triboelectricity generation by a sliding Schottky nanocontact on $\mathrm{MoS}_{2}$ multilayers, Nat. Nanotechnol. 13, 112 (2018).

[8] David C. Coffey, Obadiah G. Reid, Deanna B. Rodovsky, Glenn P. Bartholomew, and David S. Ginger, Mapping local photocurrents in polymer/fullerene solar cells with photoconductive atomic force microscopy, Nano Lett. 7, 738 (2007).

[9] Fatemeh Barati, Max Grossnickle, Shanshan Su, Roger K. Lake, Vivek Aji, and Nathaniel M. Gabor, Hot carrierenhanced interlayer electron-hole pair multiplication in 2D semiconductor heterostructure photocells, Nat. Nanotechnol. 12, 1134 (2017).

[10] Gaute Otnes and Magnus T. Borgström, Towards high efficiency nanowire solar cells, Nano Today 12, 31 (2017).

[11] J. A. Galvis, E. Herrera, C. Berthod, S. Vieira, I. Guillamón, and H. Suderow, Tilted vortex cores and superconducting gap anisotropy in $2 \mathrm{H}-\mathrm{NbSe}_{2}$, Commun. Phys. 1, 30 (2018).

[12] Dmitry Mikulik, Maria Ricci, Gozde Tutuncuoglu, Federico Matteini, Jelena Vukajlovic, Natasa Vulic, Esther Alarcon-Llado, and Anna Fontcuberta i Morral, Conductiveprobe atomic force microscopy as a characterization tool for nanowire-based solar cells, Nano Energy 41, 566 (2017).

[13] Jiangyu Li, Boyuan Huang, Ehsan Nasr Esfahani, Linlin Wei, Jianjun Yao, Jinjin Zhao, and Wei Chen, Touching is believing: Interrogating halide perovskite solar cells at the nanoscale via scanning probe microscopy, npj Quantum Mater. 2, 56 (2017).

[14] Jeremy Hieulle, Collin Stecker, Robin Ohmann, Luis K. Ono, and Yabing Qi, Scanning probe microscopy applied to organic-inorganic halide perovskite materials and solar cells, Small Methods 2, 1700295 (2018).

[15] S. Somnath, K. J. H. Law, A. N. Morozovska, P. Maksymovych, Y. Kim, X. Lu, M. Alexe, R. Archibald, S. V. Kalinin, S. Jesse, and R. K. Vasudevan, Ultrafast current imaging by Bayesian inversion, Nat. Commun. 9, 513 (2018).

[16] Alexei Belianinov, Sergei V. Kalinin, and Stephen Jesse, Complete information acquisition in dynamic force microscopy, Nat. Commun. 6, 6550 (2015).

[17] Intermodulation Products AB, https://intermodulation-prod ucts.com/, [Accessed:03-December-2018].

[18] F. Giannazzo, S. Sonde, V. Raineri, and E. Rimini, Screening length and quantum capacitance in graphene by scanning probe microscopy, Nano Lett. 9, 23 (2009).

[19] S. Ilani, L. A. K. Donev, M. Kindermann, and P. L. McEuen, Measurement of the quantum capacitance of interacting electrons in carbon nanotubes, Nat. Phys. 2, 687 (2006).

[20] Hyunah Kwon, Jun-Sik Yoon, Yuna Lee, Dong Yeong Kim, Chang-Ki Baek, and Jong Kyu Kim, An array of metal oxides nanoscale hetero $p-n$ junctions toward designable and highly-selective gas sensors, Sens. Actuators B: Chem. 255, 1663 (2018).

[21] Isabella Concina, Zafar Hussain Ibupoto, and Alberto Vomiero, Semiconducting metal oxide nanostructures for water splitting and photovoltaics, Adv. Energy Mater. 7, 1700706 (2017).

[22] Sven Rühle, Assaf Y. Anderson, Hannah-Noa Barad, Benjamin Kupfer, Yaniv Bouhadana, Eli Rosh-Hodesh, and Arie Zaban, All-oxide photovoltaics, J. Phys. Chem. Lett. 3, 3755 (2012).

[23] Hyo-Min Kim, Jeonggi Kim, Sin-Young Cho, and Jin Jang, Solution-processed metal-oxide $p-n$ charge generation junction for high-performance inverted quantumdot light-emitting diodes, ACS Appl. Mater. Interfaces 9, 38678 (2017).

[24] James W. Cooley and John W. Tukey, An algorithm for the machine calculation of complex Fourier series, Math. Comput. 19, 297 (1965).

[25] Eric Jones, Travis Oliphant, Pearu Peterson et al., SciPy: Open source scientific tools for Python, (2001), [online, available at http://www.scipy.org/]. 